\title{
The Effect of Fixed Implant Prosthodontic \\ Treatment on Mastication Predominance in Patients with Unilateral Posterior Missing Teeth
}

IMPLANT THERAPY OUTCOMES, PROSTHETIC ASPECTS
AUTHORS: ○KYOSUKE OKI, RIKA KUWATSURU, YOSHIHIRO TSUKIYAMA, YO YAMASAKI,

YOSHIKO MATSUMOTO, KIYOSHI KOYANO

Section of Implant and Rehabilitative Dentistry, Division of Oral Rehabilitation,

Faculty of Dental Science, Kyushu University, Fukuoka, Japan

\section{Abstract}

Background:

The habit of chewing predominantly on one side is called mastication predominance. Excessive sestication predominance may cause stomatognathic dysf mascion. Hence, chewing equally on both sides is generally recommended. In our previous study, we found that patients with missing posterior teeth on one side chewed on one side more predominantly than healthy dentate subjects, although there was no significant difference in masticatory efficiency.

Aim

The purpose was to investigate the effect of prosthodontic treatment for patients with unilateral effects of treatment with removal

\section{Materials and Methods:}

The sample consisted of 86 patients with unilateral posterior missing teeth who received prosthodontic treatments: removable partial denture (RPD group) or fixed implant prosthesis (IMP group). EMG activities were recorded from the bilateral masseter muscles while subjects were asked to chew chewing gum freely. The assessment of mastication predominance was conducted before and after prosthodontics treatments. The chewing side (right or left) was judged by the level of root mean square EMG amplitude. The mastication predominance was then objectively assessed by mastication predominant index (MPI): the proportion in the number of the difference between the right and left

Atter cluster evaluation of 70 healthy dentate patients, $40 \%$ of MPI was applied to out cut off value. Based on the criteria, MPI before and after treatment was evaluated.

Results

The groups. $(\mathrm{P}<0.05)$

When judging, MPI over $40 \%$ turned into under $40 \%$ by prosthodontic treatment as significant Whe free-end missing unilateral posterior region $(P<0.05)$.

\section{Conclusion:}

Masticatory predominance was improved by prosthodontics treatments for patients with missin teeth in the unilateral posterior region. Especially in the implant prosthesis group, significant inprovement of masticatory predominance was observed compared to the removable partial denture group for the patients of free-end missing unilateral posterior region.

\section{Background and Aim}

Mastication predominance

Habits/ behavior chewing on

\section{Excessive mastication}

$$
\text { ( }
$$

$$
\text { Harmful on }
$$

stomatognathic system

\section{Wilding et al., 1992, Bates et al., 1975}

\section{Our previous studies}

Objective Assessment of actual chewing side by measurement of

$$
\text { bilateral masseter muscle electromyography. }
$$

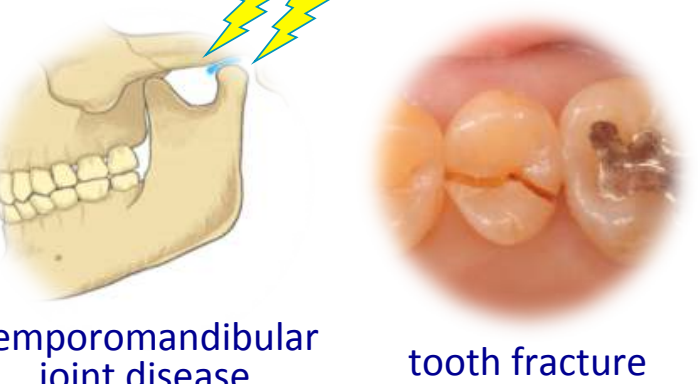

Objective assessment of mastication predominance in healthy

dentate subjects and patients with unilateral posterior missing teeth. Yamasaki et al., 2016

The individuals with missing teeth in the unilateral posterior region exhibited - tendency of chewing in the contralateral to the missing teeth side

more aware of mastication predominance than healthy dentate individuals

\section{Purpose}

1. To investigate the effect of prosthodontic treatment for patients with unilateral missing posterior teeth in terms of mastication predominance.

2. To examine the differences in the effect of treatment with

\begin{tabular}{|c|c|c|}
\hline \multirow{2}{*}{ subjects } & \multicolumn{2}{|c|}{$\begin{array}{l}86 \text { patients with missing teeth in the unilateral posterior region } \\
22 \text { men and } 64 \text { women,Mean age } 61.2(47-75) \text { years old }\end{array}$} \\
\hline & $\begin{array}{l}\text { The implant prosthesis group } \\
\text { (IP group) } n=45\end{array}$ & $\begin{array}{l}\text { The removable partial denture } \\
\text { group (RPD group) } n=41\end{array}$ \\
\hline
\end{tabular}
removable partial dentures and implant supported fixed prosthesis.

\section{Methods and Materials}

\section{Inclusion - Over 20 years old}

criteria - Natural teeth or proper fixed prosthesis in the remaining teeth region

Exclusion - Wearing teeth-supported or implant-supported overdentures

criteria - Existing systemic and/or dental diseases that influence on mastication

criteria - Difficulty cooperation in the study, such as dementia

Recording of EMG activity

MG activities record from the bilateral masseter muscles while subjects chewed test foods freely Test foods: one stick of chewing gum

prosthesis treatment and prosthesis treatment after 1 month later

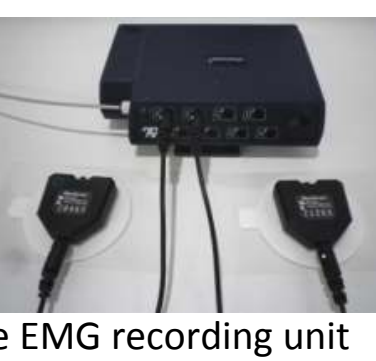

SFREEZONE Chewing gum: 18×18×4mm FREE ZONE
Analysis of EMG activity

EMG signals were converted to the root mean square (RMS) values

The number of right- and left-side strokes was counted from the EMG data of chewing test food

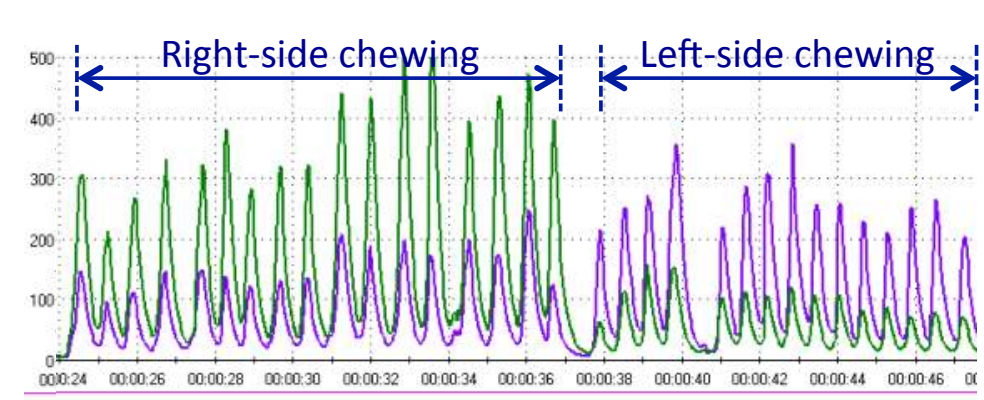

Assessment of mastication predominance

Mastication Predominance Score (MPS) and Mastication Predominance Index (MPI) was calculated by the following formula

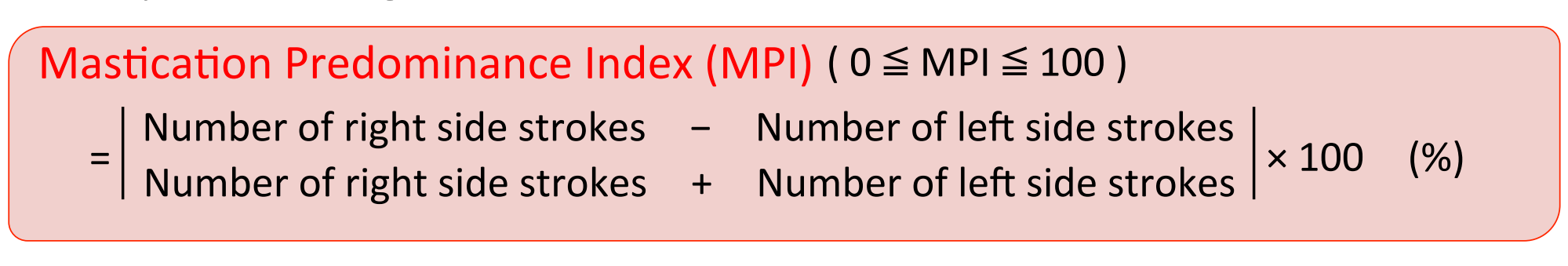

\section{Results}

【Criteria for evaluating mastication predominance】
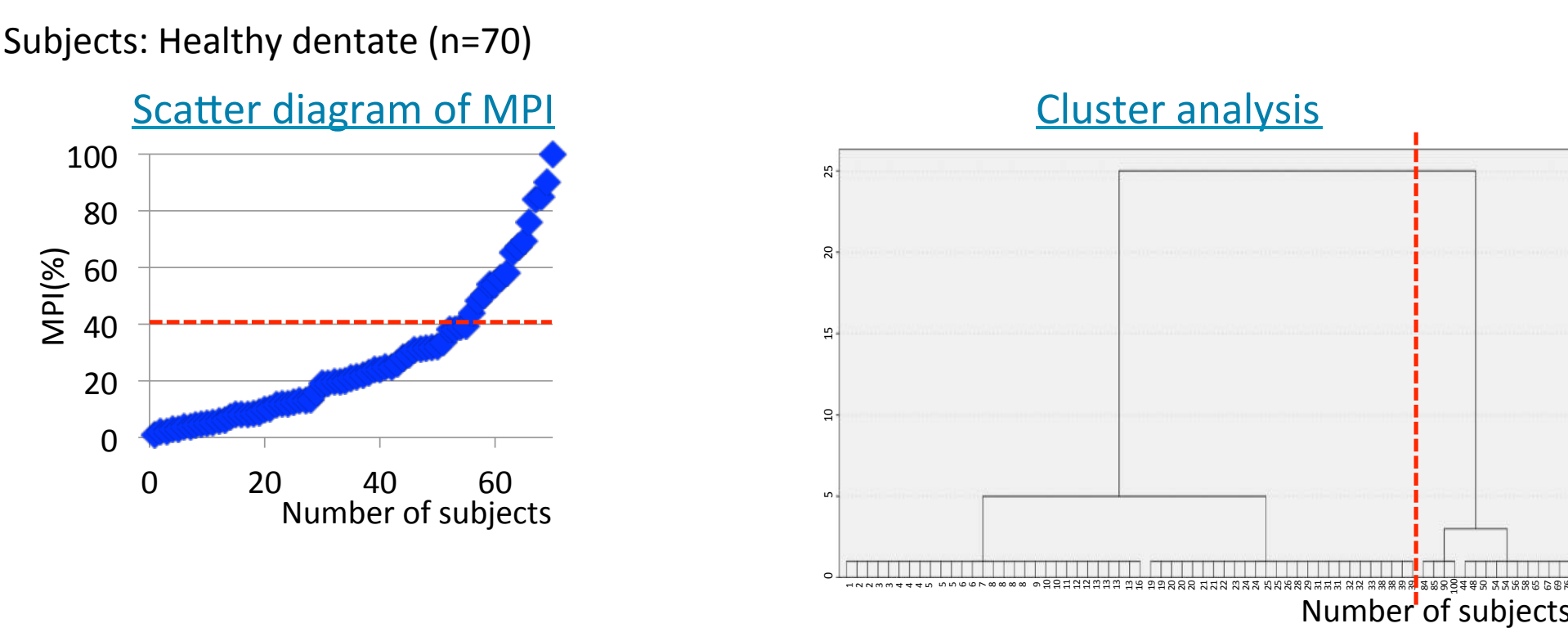

MPI $<0$ 39\% : the no existence of mastication predominance (NMP)

$\mathrm{MPI} \geqq 40 \sim 100 \%$ : the existence of mastication predominance predominance (EMP)
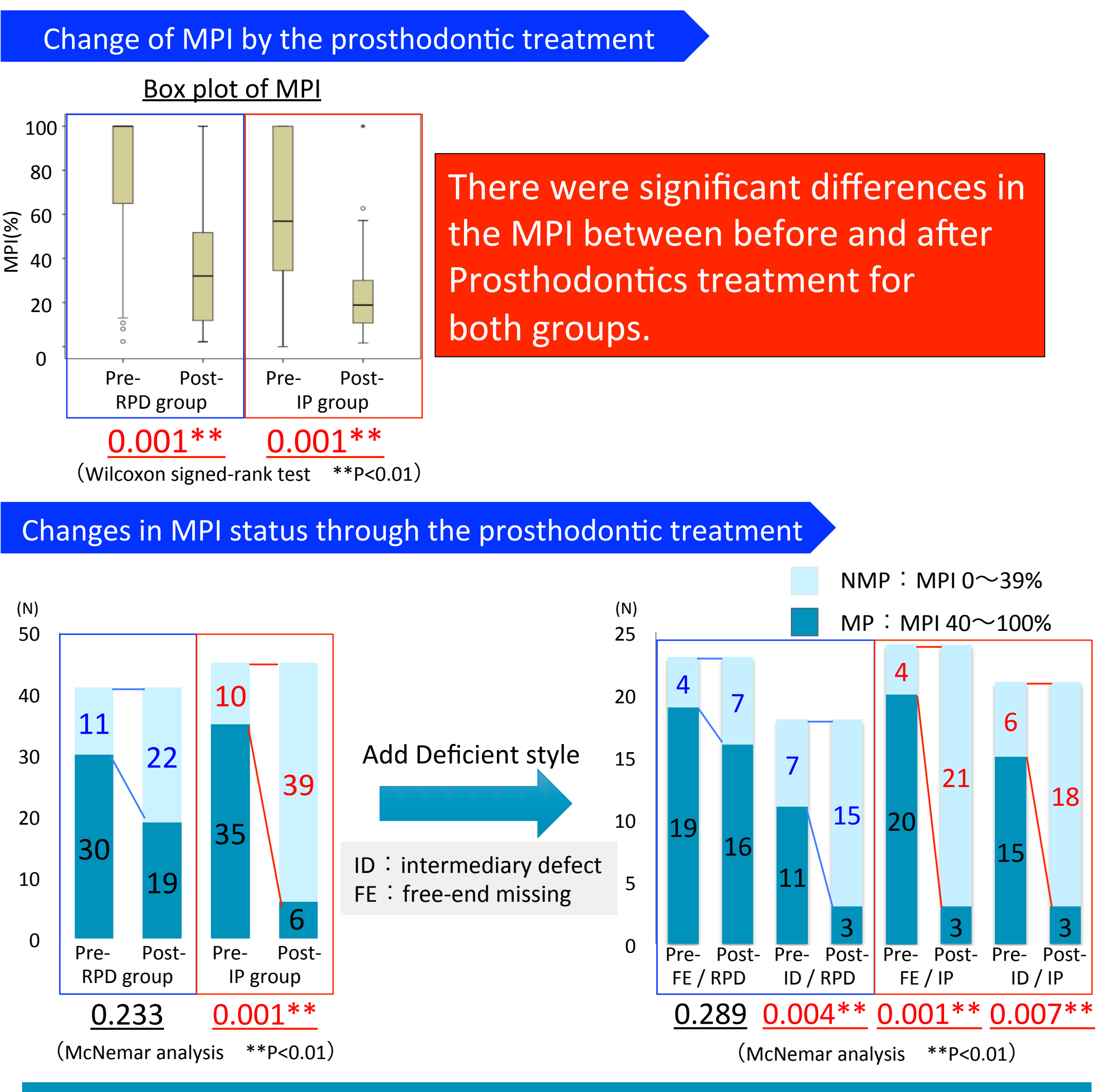

Conclusions

1. Masticatory laterality was improved by prosthodontic treatments for patients with missing teeth in the unilateral posterior region.

2. Especially in the implant prosthesis group, significant improvement of masticatory predominance was observed compared to the removable partial denture group for the patients of free-end missing unilateral posterior region.

\section{References}

Iwashita H, Tsukiyama Y, Kori H, Kuwatsuru R, Yamasaki Y, Koyano K. Comparative cross-sectional study of masticatory performance and mastication predominance for patients with missing posterior teeth. J Prosthodont Res. 2014;

Yamasaki Y, Kuwatsuru R, Tsukiyama Y, Matsumoto H, Oki K, Koyano K. Objective assessment of actual chewing side by measurement of bilateral masseter muscl electromyography. 2015;

Yamasaki Y, Kuwatsuru R, Tsukiyama Y, Oki K, Koyano K. Objective assessment of mastication predominance in healthy dentate subjects and patients with unilatera
posterior missing teeth. J Oral Rehabil. 2016; 